\title{
Synergism between Tobramycin and Ceftazidime against a Resistant Pseudomonas aeruginosa Strain, Tested in an In Vitro Pharmacokinetic Model
}

\author{
JAN G. DEN HOLLANDER, ${ }^{1 *}$ ALPHONSUS M. HORREVORTS, ${ }^{2}$ MARY-LOU P. J. VAN GOOR, ${ }^{3}$ \\ HENRI A. VERBRUGH, ${ }^{1,3}$ AND JOHAN W. MOUTON ${ }^{1,3}$ \\ Department of Clinical Microbiology and Antimicrobial Therapy, Erasmus University Rotterdam, ${ }^{3}$ and Department of \\ Bacteriology, University Hospital Rotterdam Dijkzigt, ${ }^{1}$ Rotterdam, and Department of Medical Microbiology, \\ Red Cross and Juliana Children's Hospital, The Hague, ${ }^{2}$ The Netherlands
}

Received 25 June 1996/Returned for modification 21 August 1996/Accepted 1 November 1996

\begin{abstract}
Synergism between two antibiotics is usually tested by a checkerboard titration technique, or by time-kill methods. Both methods have the disadvantage that synergism is determined at constant concentrations of the antibiotics, which do not reflect reality in vivo. In the present study we determined whether synergism between tobramycin and ceftazidime can be found at declining concentrations below the MIC, and whether change in dosing sequence of the antibiotics would result in differences in killing. Three monotherapy and six combination therapy schedules were tested in an in vitro pharmacokinetic model, using a Pseudomonas aeruginosa resistant to both antibiotics. During all q8h dosing schedules the peak concentration $\left(C_{\max }\right)$ was adjusted to the MIC for the strain of both antibiotics. During all monotherapy regimens bacterial growth was present, while all six combination therapy schedules showed significant killing. At $t=24 \mathrm{~h}$ there were no differences between all combination therapy schedules, but at $t=8 \mathrm{~h}$ the two combination therapy schedules with administration of tobramycin once daily showed a significantly faster killing. By using the area under the killing curve (AUKC) as a parameter for synergistic killing, simultaneous combination therapy starting with tobramycin once daily was significantly better than all other regimens. We conclude that there is synergism between tobramycin and ceftazidime at declining antibiotic concentrations below the MIC, resulting in a pronounced killing of a resistant Pseudomonas strain. Infections due to resistant Pseudomonas strains could possibly be treated by a synergistic combination of these drugs.
\end{abstract}

Infections caused by Pseudomonas aeruginosa continue to pose a therapeutic dilemma because of the high rates of morbidity and mortality, and the possibility of drug resistance developing during therapy. Especially in patients with cystic fibrosis emergence of antibiotic-resistant Pseudomonas aeruginosa strains is observed and it is associated with the frequent requirement for antibiotic therapy in these patients (22). One way to overcome this problem is to use combination therapy. Both in animal models $(14,15,19,27)$ and in clinical therapy $(16,17)$ treatment with a combination of an aminoglycoside with a $\beta$-lactam has shown increased efficacy. Such combination therapy is generally used to increase bactericidal activity and/or the rate of killing in vivo, and to prevent the emergence of drug resistance. Combination therapy is also used to broaden the antimicrobial spectrum in critically ill patients while awaiting a bacteriologic diagnosis or because patients have suspected or proven polymicrobial infections (8). The increased clinical response to combination therapy is usually explained to be due to synergism between the antibiotics used. Synergism of a combination of antibiotics can be expressed as fractional inhibitory concentration indices (FICi) derived from a checkerboard titration $(2,6,11,26)$. Another way to detect synergism is by performing time kill curve studies $(11,28)$. However, these methods only use constant concentrations of the antibiotics, which do not take dynamically changing concentrations into account, as seen in humans. It

* Corresponding author. Mailing address: Dept. Bacteriology, L327, University Hospital Rotterdam Dijkzigt, Dr. Molewaterplein 40, 3015 GD Rotterdam, The Netherlands. Phone: 31-(0)10-4636840. Fax: 31(0)10-4633875. has been suggested that synergism should be investigated using time kill experiments determined in in vitro models that can simulate human pharmacokinetics $(1,3,31)$.

The purpose of the present study was twofold: (i) to determine whether synergism was present between tobramycin and ceftazidime against a strain of Pseudomonas aeruginosa that is resistant to both antibiotics, during declining antibiotic concentrations, thus resulting in killing at concentrations below the MIC, and (ii) whether different dosing regimens of the antibiotics result in differences in the rate of killing.

\section{MATERIALS AND METHODS}

Bacterial strain and media. The strain used for this experiment was Pseudomonas aeruginosa CF 133, a non-mucoid strain, isolated from sputum of a cystic fibrosis patient. The MIC was $16 \mathrm{mg} /$ liter for tobramycin and $64 \mathrm{mg} / \mathrm{liter}$ for ceftazidime, as determined by standard broth macrodilution method in supplemented Mueller-Hinton broth and by agar dilution method on ISO sensitest aga (ISO; Oxoid, Basingstoke, Hampshire, England) (24), using P. aeruginosa ATCC 27853 as a test strain. The mechanism of resistance for aminoglycosides was partly determined by identification of the aminoglycoside-modifying enzymes as described by Van de Klundert et al. (30). The mechanism of resistance for $\beta$-lactam antibiotics was determined by semi-quantitative susceptibility testing, substrate analysis and iso-electric focusing of the extracted $\beta$-lactamase (29). Mueller-Hinton broth (Difco, Amsterdam, The Netherlands) supplemented with $\mathrm{Ca}^{2+}(25 \mathrm{mg} /$ liter $)$ and $\mathrm{Mg}^{2+}(12.5 \mathrm{mg} /$ liter $)$ (MHBs) was used in all experiments. All bacterial samples were plated on Tryptone soy agar (TSA; Oxoid, Basingstoke, Hampshire, England).

Antibiotics. Tobramycin was obtained from Eli Lilly and Company (Nieuwegein, The Netherlands) and ceftazidime was obtained from Glaxo (Zeist, The Netherlands). Stock solutions were prepared according to the guidelines of the National Committee for Clinical Laboratory Standards (24).

FIC indices. Fractional inhibitory concentration indices (FICi) were determined by a modified dilution checkerboard macrotitration technique (12) with synergism defined as a FICi of $\leq 0.8$ (13). When two-fold dilution series were used synergism was defined as a FICi $\leq 0.5$ (28). FICs and FICi were calculated in the usual way $(2,7)$. 
Time-kill curves. Time-kill curves were performed with tobramycin and ceftazidime alone and in combination at concentrations of $0.125,0.25,0.5,1$, and 2 times the MIC in shaking tubes containing $10 \mathrm{ml}$. For each experiment a fresh overnight culture was made in $\mathrm{MHBs}$ at $37^{\circ} \mathrm{C}$, and then diluted in prewarmed MHBs and shaken for $2 \mathrm{~h}$, resulting in a starting inoculum of approximately $5 \times$ $10^{5} \mathrm{CFU} / \mathrm{ml}$. Samples of this logarithmic culture were diluted with prewarmed antibiotic solutions containing two times the final antibiotic concentration, or for the control pure MHBs. Samples were taken at $0,1,2,4,6$, and $24 \mathrm{~h}$ of incubation. The numbers of $\mathrm{CFU} / \mathrm{ml}$ were determined after making appropriate dilutions in cold saline, and $0.1 \mathrm{ml}$ was plated on TSA plates, and incubated overnight at $37^{\circ} \mathrm{C}$. All time-kill curves were performed in duplicate. Synergism in time-kill methods is defined as a decrease $\geq 2 \log _{10} \mathrm{CFU} / \mathrm{ml}$ in the combination regimen compared to the best monotherapy regimen (28). The area under the bacterial killing curve $\left(\mathrm{AUKC}_{0-24 \mathrm{~h}}\right)$ was calculated by using the trapezoidal rule on logarithmically transformed observed data points.

In vitro pharmacokinetic model. The model used was described in detail previously (21). Briefly, a two-compartment model consisting of one central compartment and three peripheral compartments (disposable dialyzer units [ST23; Baxter, Utrecht, The Netherlands]) was used to expose the bacteria in the peripheral compartments to changing antibiotic concentrations, mimicking human pharmacokinetics. At $t=0 \mathrm{~h}$ the peripheral compartments were inoculated with a logarithmic culture of $P$. aeruginosa $\mathrm{CF} 133$ of approximately $5 \times 10^{5}$ $\mathrm{CFU} / \mathrm{ml}$, prepared separately for each compartment as described for the timekill method. Control growth curves of $P$. aeruginos $\mathrm{CF} 133$ in the in vitro model were determined the same way only without adding antibiotics.

Dosing regimens. The following dosing regimens were tested over $24 \mathrm{~h}$ : tobramycin monotherapy $\mathrm{q} 8 \mathrm{~h}$ daily and once daily, ceftazidime monotherapy $\mathrm{q} 8 \mathrm{~h}$ daily, combination therapy of tobramycin $\mathrm{q} 8 \mathrm{~h}$ and ceftazidime $\mathrm{q} 8 \mathrm{~h}$ at indicated time intervals, $t=0,8$, and $16 \mathrm{~h}$ and $t=20 \mathrm{~min}, 8 \mathrm{~h} 20 \mathrm{~min}$, and $16 \mathrm{~h} 20 \mathrm{~min}$ (i.e., simultaneous administration), or $t=0,8$, and $16 \mathrm{~h}$ and $t=4,12$, and $20 \mathrm{~h}$ (i.e., non-simultaneous administration), and finally combination therapy of tobramycin once daily and ceftazidime q8h daily. The antibiotics were infused over 20 minutes, and the peak concentration was determined at 10 minutes after the infusion $(t=30 \mathrm{~min}$.). In the $\mathrm{q} 8 \mathrm{~h}$ daily regimen the dose was chosen to obtain a peak concentration identical to the MIC for the strain (i.e., $16 \mathrm{mg} / \mathrm{liter}$ for tobramycin and $64 \mathrm{mg} /$ liter for ceftazidime). For the once-daily tobramycin regimen the dose was chosen to obtain a peak concentration of $32 \mathrm{mg} / \mathrm{liter}(2 \times$ MIC). The half life $\left(t_{1 / 2}\right)$ for both tobramycin and ceftazidime was adjusted to 2 hours. One-milliliter samples were taken from $t=0 \mathrm{~h}$, every hour, and at $t=0.5$, $4.5,8.5,12.5,16.5$, and $20.5 \mathrm{~h}$. The samples were immediately washed (twice) with sterile cold saline and $0.1 \mathrm{ml}$ was plated on TSA plates (limit of detection = $10 \mathrm{CFU} / \mathrm{ml}$ ). Samples were assayed for tobramycin by fluorescence polarization immunoassay using TDxFLx (Abbott Diagnostic Division, Amstelveen, The Netherlands) and for ceftazidime by high-performance liquid chromatography (HPLC) as described earlier (23). The lower limit of sensitivity of the assay was $0.5 \mathrm{mg} /$ liter. The between-day between-sample variation was less than $7 \%$. Control runs were performed regularly.

MICs. MICs for the strains isolated at $t=0,8,16$, and $24 \mathrm{~h}$ were determined using a standard agar dilution method (24), to determine development of resistance during the experiment. To detect more resistant mutants in the starting inoculum, a fresh logarithmic culture of $P$. aeruginosa CF 133 was plated on ISO-agar plates containing tobramycin or ceftazidime at a concentration of 8,16 , $32,64,128$, or $256 \mathrm{mg} / \mathrm{liter}$ and $32,64,128,256,512$ or $1,024 \mathrm{mg} /$ liter, respectively.

Initial bactericidal effect. The initial killing effect of a regimen was expressed as changes in $\log _{10} \mathrm{CFU} / \mathrm{ml}$ at one fixed time point during an experiment, or as the time needed to reduce the inoculum to $10^{3} \mathrm{CFU} / \mathrm{ml}(1)$.

Statistical analysis. Peak and trough concentrations, half-life of the antibiotics during the different experiments and the time kill curves (i.e., the difference between the $\log _{10} \mathrm{CFU} / \mathrm{ml}$ at $t=0 \mathrm{~h}$ and $t=8$, and $t=0$ and $24 \mathrm{~h}$, the time to a $10^{3} \mathrm{CFU} / \mathrm{ml}$ reduction of the inoculum) were compared by using a two-way analysis of variance (ANOVA) and Tukey's test for multiple comparison of significance. For analysis of the AUKC, time kill curves were compared with each other using a two-way analysis of variance (ANOVA) for repeated measurements. Monotherapy was tested against combination therapy, combination therapy regimens were tested against each other, and single-dose tobramycin was tested against multiple-dose tobramycin. The Instat 2 computer package (9) was used for all statistical analysis. A $P$ value of 0.05 two-tailed was considered significant.

\section{RESULTS}

Mechanism of resistance. The $P$. aeruginosa $\mathrm{CF} 133$ produced aminoglycoside-modifying enzymes, which were identified as AAC (6') II and APH (3'), and produced a $\beta$-lactamase which was identified as a stably depressed chromosomal encoded class I $\beta$-lactamase (4).

FIC indices. The FICi of $P$. aeruginosa CF 133 for tobramycin and ceftazidime, determined using a two-fold dilution method for the checkerboard titration, was 0.55 , indicating

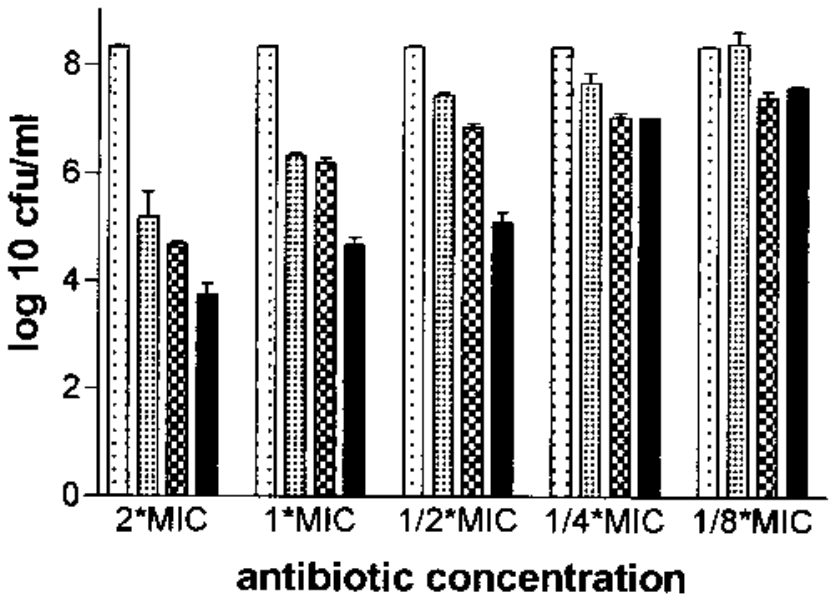

FIG. 1. Difference in bactericidal effect after $24 \mathrm{~h}$ exposure to tobramycin (ख) or ceftazidime ( $\mathbf{E}: \mathbf{\Xi}$ ) alone and in combination $(\boldsymbol{D})$ at a concentration range of $2 \times$ MIC to $1 / 8 \times$ MIC. Control growth curve (--- ). Data are means $\pm \mathrm{SD}$ of two experiments of the $\mathrm{CFU} / \mathrm{ml}$ present at $24 \mathrm{~h}$.

indifference. However, if the modified macrotitration technique was used (12), the FICi was 0.37 , indicating synergism between tobramycin and ceftazidime for this strain (13).

Time-kill curves. Numbers of $\log _{10} \mathrm{CFU} / \mathrm{ml}$ of single agent exposure and combination agent exposure at different antibiotic concentrations at the end-point $(t=24 \mathrm{~h})$ are shown in Fig. 1. Combination therapy showed a significant increase in killing over monotherapy if the concentrations were $\geq$ one-half MIC, but this effect was not large enough to label it synergism according to the definition.

The $\mathrm{AUKC}_{0-24 \mathrm{~h}}$ calculated for all killing curves are shown in Table 1. For each concentration the $\mathrm{AUKC}_{0-24 \mathrm{~h}}$ of the best monotherapy regimen was compared to the $\mathrm{AUKC}_{0-24 \mathrm{~h}}$ combination therapy. All combinations at concentrations $\leq$ one MIC showed a significantly smaller $\mathrm{AUKC}_{0-24 \mathrm{~h}}$ than monotherapy (Table 1 ). At $2 \times$ MIC no significant difference between tobramycin monotherapy and combination therapy was found, probably due to the pronounced killing of tobramycin, when it is used above the MIC as in this experiment.

Pharmacokinetic data. The peak and trough concentrations of tobramycin and ceftazidime and the $t_{1 / 2}$ obtained in the in vitro model during the different dosing regimens showed no significant differences. The peak concentrations were $13.8 \pm$ $1.2 \mathrm{mg} /$ liter, $33.7 \pm 2.0 \mathrm{mg} /$ liter, and $53.1 \pm 5.3 \mathrm{mg} /$ liter, and the trough concentrations were $1.7 \pm 0.5 \mathrm{mg} /$ liter, $0.1 \pm 0.1$

TABLE 1. Bactericidal activity of tobramycin and ceftazidime given alone or in combination towards a tobramycin and ceftazidime resistant $P$. aeruginosa, expressed as the $\mathrm{AUKC}_{0-24 \mathrm{~h}}( \pm \mathrm{SD})$ determined in conventional time-kill experiments

\begin{tabular}{lcccc}
\hline \multirow{2}{*}{$\begin{array}{c}\text { Antibiotic } \\
\text { concentration }\end{array}$} & $\begin{array}{c}\text { Tobramycin } \\
\text { monotherapy }\end{array}$ & $\begin{array}{c}\text { Ceftazidime } \\
\text { monotherapy }\end{array}$ & $\begin{array}{c}\text { Combination } \\
\text { therapy }\end{array}$ & $P$ value $^{a}$ \\
\hline $2 \times$ MIC & $93 \pm 5$ & $102 \pm 2$ & $77 \pm 7$ & 0.131 \\
$1 \times$ MIC & $126 \pm 4$ & $122 \pm 3$ & $89 \pm 3$ & 0.008 \\
$1 / 2 \times$ MIC & $148 \pm 1$ & $141 \pm 1$ & $95 \pm 1$ & 0.001 \\
$1 / 4 \times$ MIC & $159 \pm 5$ & $149 \pm 4$ & $123 \pm 1$ & 0.012 \\
$1 / 8 \times$ MIC & $173 \pm 4$ & $156 \pm 1$ & $140 \pm 1$ & 0.001 \\
\hline
\end{tabular}

${ }^{a} P$ value obtained by comparing the best single agent exposure to the combination agent exposure. 

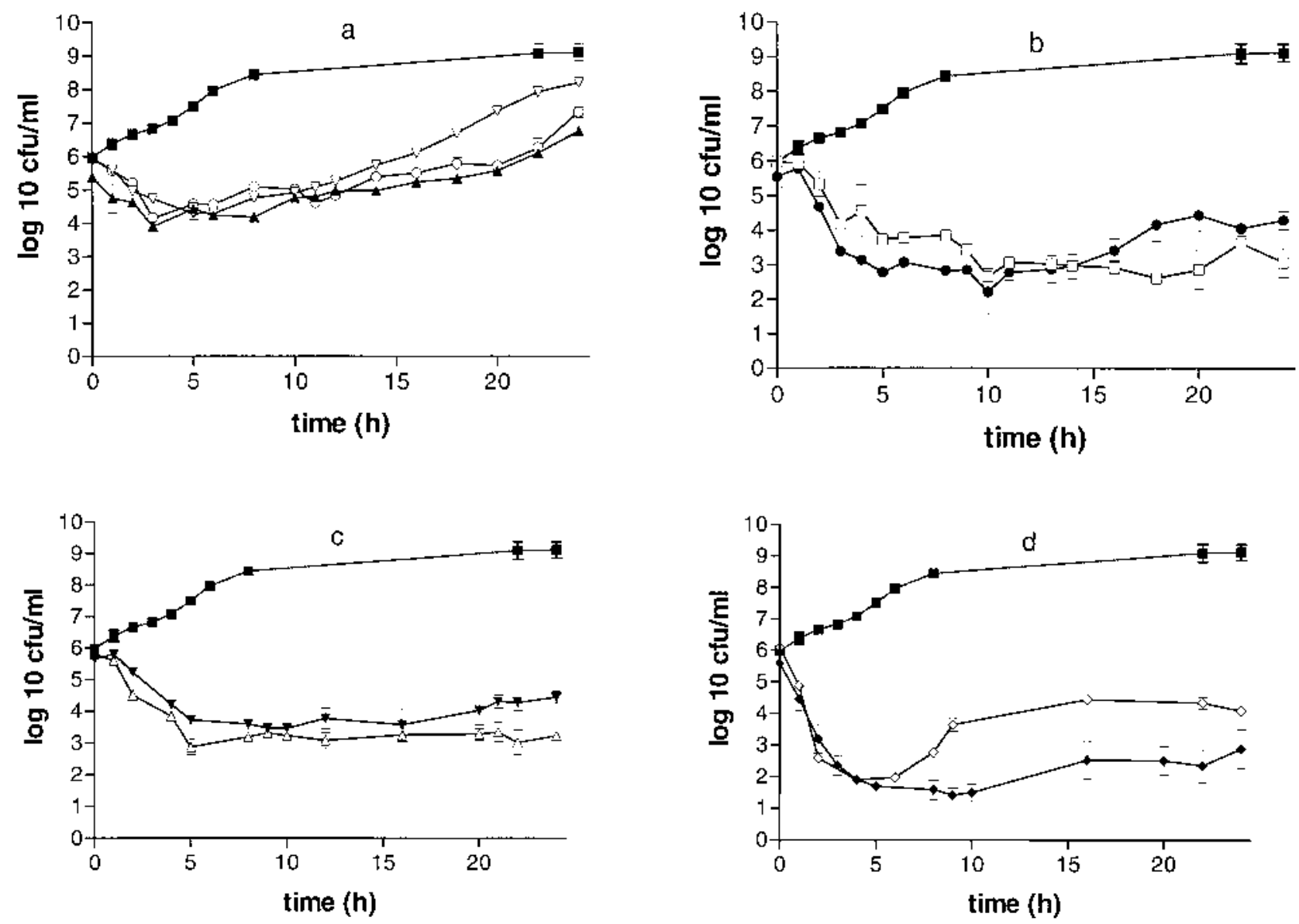

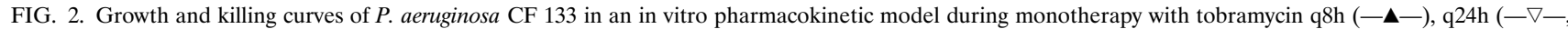
and ceftazidime $\mathrm{q} 8 \mathrm{~h}(-\bigcirc-)$ (a) and during combination therapy regimens tobramycin (q8h) at $t=0 \mathrm{~h}$ and ceftazidime (q8h) at $t=20 \mathrm{~min}$ ( $-\square-$ ), and vice versa $(--)$ (b), tobramycin (q8h) at $t=0 \mathrm{~h}$ and ceftazidime (q8h) at $t=4 \mathrm{~h}(-\nabla-)$ ), and vice versa $(-\triangle-)$ (c), and tobramycin (q24h) at $t=0 \mathrm{~h}$ and ceftazidime (q8h) at $t=20 \mathrm{~min}(--$, and vice versa $(-\diamond-)(\mathrm{d})$. Data are geometric means \pm SD of two separate experiments, performed in triplicate. $-\square$ in all panels denotes the control growth curve of the test strain in absence of antibiotics.

$\mathrm{mg} / \mathrm{liter}$, and $5.1 \pm 1.1 \mathrm{mg} /$ liter for tobramycin (q8h), tobramycin (q24h), and ceftazidime (q8h), respectively. The $t_{1 / 2}$ was $2.3 \pm 0.2 \mathrm{~h}$, which is slightly but not significantly higher than the intended half-life. The half-lives for both drugs were not significantly different.

Growth curve and monotherapy. Monotherapy with tobramycin ( $\mathrm{q} 8 \mathrm{~h}$ and $\mathrm{q} 24 \mathrm{~h}$ ) and ceftazidime (q8h) showed an initial decrease in $\mathrm{CFU} / \mathrm{ml}$ of this resistant strain to approximately $10^{4} \mathrm{CFU} / \mathrm{ml}$ during the first hours of treatment, followed by a bacterial regrowth (Fig. 2a). There was no significant difference between the effect of the three monotherapy dosing regimens determined by comparing the $\Delta \log _{10} \mathrm{CFU} / \mathrm{ml}$ at $t=8$ and $24 \mathrm{~h}$ (Table 2). All monotherapy regimens showed a slight growth retardation compared to the control growth curve.

TABLE 2. Bactericidal activity of tobramycin and ceftazidime given alone or in combination for a tobramycin and ceftazidime resistant strain of $P$. aeruginosa, in an in vitro pharmacokinetic model

\begin{tabular}{|c|c|c|c|}
\hline \multirow{2}{*}{ Dosing regimen } & \multicolumn{2}{|c|}{$\Delta \log _{10} \mathrm{CFU} / \mathrm{ml}^{a}$} & \multirow{2}{*}{$\begin{array}{l}\text { Time to reduce the inoculum } \\
\left(5 \times 10^{5} \mathrm{CFU} / \mathrm{ml}\right) \text { to } \\
<10^{3} \mathrm{CFU} / \mathrm{ml}(\mathrm{h})\end{array}$} \\
\hline & $t=8 \mathrm{~h}$ & $t=24 \mathrm{~h}$ & \\
\hline Tobramycin q8h & $-1.2 \pm 0.1$ & $1.4 \pm 0.1$ & $\infty$ \\
\hline Tobramycin q24h & $-1.1 \pm 0.1$ & $2.3 \pm 0.4$ & $\infty$ \\
\hline Ceftazidime q8h & $-0.8 \pm 0.1$ & $1.4 \pm 0.3$ & $\infty$ \\
\hline Tobramycin ( $\mathrm{q} 8 \mathrm{~h})$ at $t=0 \mathrm{~h} /$ ceftazidime $(\mathrm{q} 8 \mathrm{~h})$ at $t=20 \min$ & $-2.1 \pm 0.1$ & $-2.9 \pm 0.8^{b}$ & $9.6 \pm 0.4$ \\
\hline Ceftazidime (q8h) at $t=0 \mathrm{~h} /$ tobramycin ( $88 \mathrm{~h})$ at $t=20 \mathrm{~min}$ & $-2.7 \pm 0.5$ & $-1.3 \pm 1.0^{b}$ & $4.8 \pm 0.2$ \\
\hline Tobramycin (q8h) at $t=0 \mathrm{~h} /$ ceftazidime $(\mathrm{q} 8 \mathrm{~h})$ at $t=4 \mathrm{~h}$ & $-2.1 \pm 0.1$ & $-1.2 \pm 0.3^{b}$ & $8.3 \pm 0.5$ \\
\hline Ceftazidime (q $8 \mathrm{~h})$ at $t=0 \mathrm{~h} /$ tobramycin (q $8 \mathrm{~h})$ at $t=4 \mathrm{~h}$ & $-2.6 \pm 0.2$ & $-2.6 \pm 0.3^{b}$ & $9.9 \pm 4.7$ \\
\hline Tobramycin (q24h) at $t=0 \mathrm{~h} /$ ceftazidime (q8h) at $t=20 \min$ & $-3.9 \pm 0.8^{b}$ & $-2.6 \pm 1.3^{b}$ & $2.2 \pm 0.6$ \\
\hline Tobramycin (q24h) at $t=20 \mathrm{~min} /$ ceftazidime (q8h) at $t=0 \mathrm{~h}$ & $-3.3 \pm 0.3^{b}$ & $-2.0 \pm 0.3^{b}$ & $1.7 \pm 0.1$ \\
\hline
\end{tabular}

${ }^{a}$ Data are means \pm SD of two separate experiments, performed in triplicate.

${ }^{b}$ Decrease in $\Delta \log _{10} \mathrm{CFU} / \mathrm{ml}>2 \log _{10}$, compared to the best monotherapy regimen. 


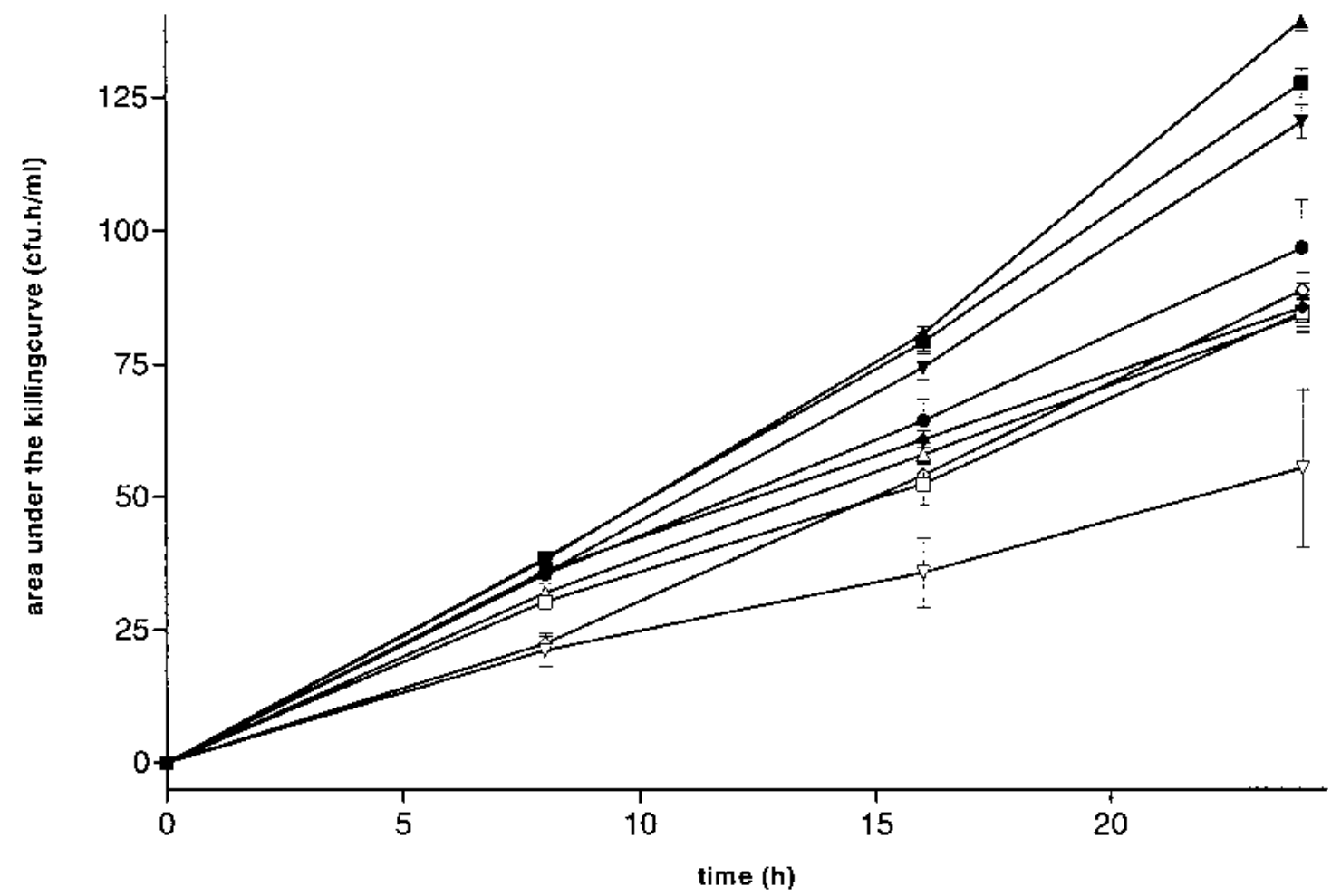

FIG. 3. Bactericidal effect of tobramycin or ceftazidime alone or in combination expressed as the cumulative area under the bacterial killing curve (AUKC). Lines represent tobramycin q8h alone $(-\boldsymbol{\nabla}-)$, tobramycin $\mathrm{q} 24 \mathrm{~h}$ alone $(-\boldsymbol{\Lambda}-)$, and ceftazidime $\mathrm{q} 8 \mathrm{~h}$ alone $(-\mathbf{-})$, combination therapies tobramycin $(\mathrm{q} 8 \mathrm{~h})$ at $t=0 \mathrm{~h}$ and ceftazidime (q8h) at $t=20 \mathrm{~min}(--)$, and vice versa (- $\square$-), tobramycin (q8h) at $t=0 \mathrm{~h}$ and ceftazidime (q8h) at $t=4 \mathrm{~h}(-\bullet-)$, and vice versa (一 $-\triangle$ ), tobramycin (q24h) at $t=0 \mathrm{~h}$ and ceftazidime (q8h) at $t=20 \mathrm{~min}(-\nabla-)$, and vice versa (- -$)$. Data are means $\pm \mathrm{SD}$ of two separate experiments performed in triplicate.

Combination therapy. All four combination therapies including $\mathrm{q} 8 \mathrm{~h}$ tobramycin showed an initial killing during the first five hours of treatment, followed by a stabilization of the colony counts around $10^{3} \mathrm{CFU} / \mathrm{ml}$ (Fig. $2 \mathrm{~b}$ and c). By comparing the $\Delta \log _{10} \mathrm{CFU} / \mathrm{ml}$ at $t=8 \mathrm{~h}$ and $24 \mathrm{~h}$, no differences were found among all combination therapy regimens. However, the $\Delta \log _{10} \mathrm{CFU} / \mathrm{ml}$ of all combination therapy regimens were significantly different from all monotherapy regimens.

Combination therapy regimens, including $\mathrm{q} 24 \mathrm{~h}$ tobramycin, showed a pronounced killing during the first three hours of treatment. When tobramycin was given before ceftazidime the colony counts stabilized around $10^{2}$ to $10^{3} \mathrm{CFU} / \mathrm{ml}$; however, if ceftazidime was given as the first agent a slight bacterial regrowth was seen to $10^{3}$ to $10^{4} \mathrm{CFU} / \mathrm{ml}$ (Fig. 2d). This difference was not significant at $t=24 \mathrm{~h}(P=0.47$, Table 2$)$.

Calculating the time needed to reduce the inoculum to $10^{3}$ $\mathrm{CFU} / \mathrm{ml}$ (Table 2) as a parameter for the initial killing effect, as described by Barclay et al. (1), demonstrated that the combination therapy regimens with tobramycin $\mathrm{q} 24 \mathrm{~h}$ had a significantly faster initial killing compared to all other combination therapy regimens $(0.001<P<0.01)$, except for the $\mathrm{q} 8 \mathrm{~h}$ combination therapy regimen with ceftazidime given at $t=0 \mathrm{~h}$ and tobramycin at $t=20 \mathrm{~min}$.

Synergism. Synergism was shown by a decrease $\geq 2 \log _{10}$ $\mathrm{CFU} / \mathrm{ml}$ compared to the best monotherapy regimen at $t=8 \mathrm{~h}$ only for the tobramycin $\mathrm{q} 24 \mathrm{~h}$ combination therapy regimens, and at $t=24 \mathrm{~h}$ for all combination therapy regimens (Table 2).

Synergism was also determined by calculation of the area under the bacterial killing curve (AUKC). An AUKC ( 0 to $8 \mathrm{~h}$, 0 to $16 \mathrm{~h}$, and 0 to $24 \mathrm{~h}$ ) was calculated for all dosing regimens.
If the AUKC was significantly smaller for a combination therapy compared to the best monotherapy regimen, it was considered as synergism. The cumulative AUKC at $t=8,16$, and $24 \mathrm{~h}$ showed that at $t=8 \mathrm{~h}$ both combination therapies using tobramycin once daily gave a significant smaller AUKC than all other regimens $(P<0.001)$ (Fig. 3). At $t=16 \mathrm{~h}$ and $t=24 \mathrm{~h}$ all combination therapy regimens show a significant smaller AUKC than all monotherapy regimens, with one combination therapy regimen being significantly better than all other regimens (tobramycin once daily at $t=0 \mathrm{~h}$, and ceftazidime thrice daily at $t=20 \mathrm{~min}, 8 \mathrm{~h} 20 \mathrm{~min}$, and $16 \mathrm{~h} 20 \mathrm{~min}(0.0001<P<$ $0.01)$ ).

Emergence of resistance. The MICs of the bacteria isolated during combination therapy regimens at $t=8,16$, and $24 \mathrm{~h}$ increased about 4 times for tobramycin, but remained $64 \mathrm{mg} /$ liter for ceftazidime. During monotherapy the MICs for the bacteria increased about four times for tobramycin and ceftazidime. No statistical difference in MIC rise was found between the dosing regimens. No resistant mutants could be detected in the logarithmic culture of the starting inoculum as used in all experiments.

\section{DISCUSSION}

The main purpose of this study was to determine whether a combination of tobramycin and ceftazidime, with peak concentrations in the range of the MIC and at declining concentrations over time, resulted in synergism. This means that the concentration was below the MIC for both agents during the complete experiment. When determining synergism, at least 
one of the drugs should have a concentration which does not affect the bacterial growth (8). The only experiment where the peak concentration exceeded the MIC was when tobramycin was given once daily. This protocol thus provided a good circumstance to demonstrate synergism. If killing was observed this can only be explained as being due to synergism, because at concentrations below the MIC, growth can be expected when only monotherapy is given. The results indicate that synergism is present at $24 \mathrm{~h}$ for all tested combination regimens of tobramycin and ceftazidime, as shown by a decrease $\geq 2 \log _{10} \mathrm{CFU} / \mathrm{ml}$ compared to all monotherapy regimens (Table 2). While growth occurred during all monotherapy experiments (Fig. 2a), the final $\mathrm{CFU} / \mathrm{ml}$ in combination therapy always was below the starting inoculum.

The choice of combinations of antimicrobial agents in therapy is often based on the MIC, and sometimes on a possible synergism demonstrated by determination of the FICi in a standard checkerboard titration, or by time kill curves. The FICi of the Pseudomonas strain used in the experiments was 0.55 when using a two-fold dilution checkerboard titration method, indicating indifference between tobramycin and ceftazidime. However when using the modified dilution checkerboard titration (12), the FICi was 0.37 , indicating a high degree of synergism between tobramycin and ceftazidime. This confirms the observations of Horrevorts et al. $(12,13)$ that the FICi determined by a modified checkerboard titration is a more sensitive technique for detecting synergism, due to the use of smaller intervals between the dilution steps. Unfortunately, studies show discordance between the results of checkerboard titrations and time-kill curve methods (25), between FICi and in vitro model studies $(20,32)$, and between FICi and clinical outcome $(5,26)$. Possibly, the simulation of declining antibiotic concentrations as can be produced in in vitro pharmacokinetic models is a better method to study synergism. Observations from these models may correlate better with clinical outcome since they are a better simulation of situations in patients.

In time kill curves, synergism is always determined at one time-point only (usually $t=24 \mathrm{~h}$ ), while synergism between the antibiotics is present during the complete experiment, and thus at each time-point. To take these dynamics into account more time points should be taken for the calculation of synergism. When using the $\mathrm{AUKC}_{0-24 \mathrm{~h}}$ as a parameter for the killing effect, all time-points during the experiments are used. Thus, the $\mathrm{AUKC}_{0-24 \mathrm{~h}}$ may be a better parameter of synergism. This method can be used for both conventional time-kill curves and for time-kill curves determined in in vitro models. By defining synergism as a significantly smaller $\mathrm{AUKC}_{0-24 \mathrm{~h}}$ of the combination therapy compared to the best monotherapy regimen, all combination therapy regimens showed synergism. However, by using the significant differences in $\mathrm{AUKC}_{0-24 \mathrm{~h}}$ only, one does not differentiate between synergism and additivity (or indifference). But it is questionable if such differentiation is clinically relevant. When combination therapy results in a significantly increased killing compared to monotherapy, combination therapy could be justified, even if there is only additivity. In clinical practice knowledge about killing during the complete time period of treatment may well be more important than just at a single time point $(t=24 \mathrm{~h})$.

The significantly faster initial killing as seen in the combination regimens with tobramycin once daily can be explained by a fast initial killing of the bacteria susceptible to tobramycin since in this case the concentration remained above the MIC for about two hours. But this fast initial killing cannot be fully explained by this high peak concentration, since it was not seen in the tobramycin once daily monotherapy regimen. Thus this fast initial killing has to be at least in part a result of the synergism of the combined agents.

During all combination therapy regimens only emergence of resistance for tobramycin was shown. The MICs of ceftazidime did not change during combination therapy. During monotherapy however, the MICs of both tobramycin and ceftazidime increased about four times. In the starting inoculum no resistant mutants could be detected by plating on an antibiotic containing agar. This indicates that combination therapy may prevent emergence of resistance.

Barclay et al. (1) described a study investigating simultaneous and nonsimultaneous infusions of gentamicin and ceftazidime against three susceptible $P$. aeruginosa strains in an in vitro pharmacokinetic dilution model. Several time points were tested for infusion; however, only one injection of the antibiotics was used in those experiments. They concluded that the nonsimultaneous administration produced greater overall killing and delay in bacterial regrowth. König et al. (18) investigated the dosing regimen of gentamicin and ampicillin against $E$. coli, and later the same group published the combination of gentamicin and ticarcillin against $P$. aeruginosa (10). Both studies showed a greater overall bacterial killing and delay in bacterial regrowth in using nonsimultaneous administration given 4 hours apart. Our study could, however, not confirm this, possibly due to the fact that we used a resistant strain.

Some reservations should be made concerning the fact that only one strain was tested with one specific mechanism of resistance. It may be possible that the conclusions based on the data for the tested strain will be different for strains with other mechanisms of resistance. At present, this limits the ability to generalize the conclusions to other strains.

In conclusion, this study indicates that infections due to a resistant Pseudomonas strain, such as frequently occur in patients with cystic fibrosis, possibly can be treated with a synergistic combination of the antibiotics to each of which the strain is resistant. With $\beta$-lactam-plus-aminoglycoside combination, the best effect is possibly produced if once daily doses of the aminoglycoside are combined with multiple daily doses of the $\beta$-lactam, since this may result in the fastest initial killing rates and in the smallest $\mathrm{AUKC}_{0-24 \mathrm{~h}}$.

\section{ACKNOWLEDGMENTS}

We thank R. van Dijk and L. Overdijk for their analytical work in part of this study, and J. A. M. van de Klundert, W. H. F. Goessens, and R. Mangal for their help with the determination of the mechanism of resistance.

\section{REFERENCES}

1. Barclay, M. L., E. J. Begg, S. T. Chambers, and D. R. Boswell. 1995. Improved efficacy with nonsimultaneous administration of first doses of gentamicin and ceftazidime in vitro. Antimicrob. Agents Chemother. 39:132-136.

2. Berenbaum, M. C. 1978 . A method for testing synergy with any number of agents. J. Infect. Dis. 137:122-130.

3. Blaser, J., B. B. Stone, M. C. Groner, and S. H. Zinner. 1985. Impact of netilmicin regimens on the activities of ceftazidime-netilmicin combinations against Pseudomonas aeruginosa in an in vitro pharmacokinetic model. Antimicrob. Agents Chemother. 28:64-68.

4. Bush, K., G. A. Jacoby, and A. A. Medeiros. 1995. A functional classification scheme for $\beta$-lactamase and its correlation molecular structure. Antimicrob. Agents Chemother. 39:1211-1233.

5. Chandrasekar, P. H., L. R. Crane, and E. J. Bailey. 1987. Comparison of the activity of antibiotic combinations in vitro with clinical outcome and resistance in serious infection by Pseudomonas aeruginosa in non-neutropenic patients. J. Antimicrob. Chemother. 19:321-329.

6. Chin, N. X., and H. C. Neu. 1983. Synergy of azlocillin with aminoglycosides. J. Antimicrob. Chemother. 11(Suppl. B):33-38.

7. Elion, G. B., S. Singer, and G. H. Hitchings. 1953. Antagonists of nucleic acid derivates. VIII. Synergism in combinations of biochemically related antimetabolites. J. Biol. Chem. 208:477-488.

8. Fantin, B., and C. Carbon. 1992. In vivo antibiotic synergism: contribution of 
animal models. Antimicrob. Agents Chemother. 36:907-912.

9. GraphPad Software, Inc. 1990. Instat 2 program manual. GraphPad Software, Inc., San Diego, Calif.

10. Guggenblicher, J. P., F. Allerberger, M. P. Dierich, R. Schmitzberger, and E. Semenitz. 1988. Spaced administration of antibiotic combinations to eliminate pseudomonas from sputum in cystic fibrosis. Lancet ii:749-750.

11. Hallander, K. O., K. Dornbusch, L. Gezelius, K. Jacobson, and I. Karlsson. 1982. Synergism between aminoglycosides and cephalosporins with antipseudomonal activity: interaction index and killing curve method. Antimicrob. Agents Chemother. 22:743-752.

12. Horrevorts, A. M., C. M. de Ridder, M. C. Poot, M. J. A. de Jonge, J. E. Degener, G. Dzoljic-Danilovic, M. F. Michel, and K. F. Kerrebijn. 1987. Checkerboard titrations: the influence of the composition of serial dilutions of antibiotics on the fractional inhibitory concentration index and fractiona bactericidal concentration index. J. Antimicrob. Chemother. 19:119-125.

13. Horrevorts, A. M., M. F. Michel, and K. F. Kerrebijn. 1987. Antibiotic interaction: interpretation of fractional inhibitory and fractional bactericidal concentration indices. Eur. J. Clin. Microbiol. 4:502-503.

14. Johnson, D. E., and B. Thompson. 1986. Efficacy of single-agent therapy with azlocillin, ticarcillin, and amikacin and beta-lactam/amikacin combinations for treatment of Pseudomonas aeruginosa bacteremia in granulocytopenic rats. Am. J. Med. 80(Suppl. 5C):53-58.

15. Johnson, D. E., B. Thompson, and F. M. Calia. 1985. Comparative activities of piperacillin, ceftazidime, and amikacin alone and in all possible combinations, against experimental Pseudomonas aeruginosa infections in neutropenic rats. Antimicrob. Agents Chemother. 27:735-739.

16. Klastersky, J., and S. H. Zinner. 1982. Synergistic combinations of antibiotics in gram-negative bacillary infections. Rev. Infect. Dis. 4:294-301.

17. Klastersky, J., R. Cappel, and D. Daneau. 1972. Clinical significance of in vitro synergism between antibiotics in gram-negative infections. Antimicrob. Agents Chemother. 2:470-475.

18. König, P., J. P. Guggenblicher, E. Semenitz, and W. Foisner. 1986. Kill kinetics of bacteria under fluctuating concentrations of various antibiotics. Chemotherapy 32:44-58.

19. Lumish, R. M., and C. W. Norden. 1976. Therapy of neutropenic rats infected with Pseudomonas aeruginosa. J. Infect. Dis. 133:538-547.

20. McGrath, B. J., K. C. Lamp, and M. J. Rybak. 1993. Pharmacodynamic effects of extended dosing intervals of imipenem alone and in combination with amikacin against Pseudomonas aeruginosa in an in vitro model. Antimicrob. Agents Chemother. 37:1931-1937.
21. Mouton, J. W., and J. G. den Hollander. 1994. Killing of Pseudomonas aeruginosa during continuous and intermittent infusion of ceftazidime in an in vitro pharmacokinetic model. Antimicrob. Agents Chemother. 38:931936.

22. Mouton, J. W., J. G. den Hollander, and A. M. Horrevorts. 1993. Emergence of antibiotic resistance amongst Pseudomonas aeruginosa isolates from cystic fibrosis patients. J. Antimicrob. Chemother. 31:919-926.

23. Mouton, J. W., A. M. Horrevorts, P. H. G. Mulder, E. P. Prens, and M. F Michel. 1990. Pharmacokinetics of ceftazidime in serum and suction blister fluid during continuous and intermittent infusion in healthy volunteers. Antimicrob. Agents Chemother. 34:2307-2311.

24. National Committee for Clinical Laboratory Standards. 1990. Methods for dilution antimicrobial susceptibility tests for bacteria that grow aerobically. Approved standard M7A2. National Committee for Clinical Laboratory Standards, Villanova, Pa.

25. Norden, C. W., H. Wentzel, and E. Keleti. 1979. Comparison of techniques for measurement of in vitro synergism. J. Infect. Dis. 140:692-733.

26. Reyes, M. P., F. Smith, and A. M. Lerner. 1984. Studies of in vitro synergy between several beta-lactam and aminoglycoside antibiotics against endocarditis strains of Pseudomonas aeruginosa. J. Infect. 8:110-117.

27. Scott, R. E., and H. G. Robson. 1976. Synergistic activity of carbenicillin and gentamicin in experimental Pseudomonas bacteremia in neutropenic rats. Antimicrob. Agents Chemother. 10:646-651.

28. Stratton, C. W., and R. C. Cooksey. 1991. Susceptibility tests: special tests, p. 1153-1166. In A. Balows (ed.), Manual of clinical microbiology, 5th ed. American Society for Microbiology, Washington, D.C.

29. Van de Klundert, J. A. M., M. H. van Gestel, E. van Doorn, and R. P. Mouton. 1986. Disc diffusion test for the determination of semi-quantitative substrate profiles of $\beta$-lactamases. J. Antimicrob. Chemother. 17:471-479.

30. Van de Klundert, J. A. M., J. S. Vliegenthart, E. van Doorn, G. P. A Bongaerts, L. Molendijk, and R. P. Mouton. 1984. A simple method for identification of aminoglycoside-modifying enzymes. J. Antimicrob. Chemother. 14:339-348

31. Vergères, P., and J. Blaser. 1992. Amikacin, ceftazidime, and flucloxacillin against suspended and adherent Pseudomonas aeruginosa and Staphylococcus epidermidis in an in vitro model of infection. J. Infect. Dis. 165:281-289.

32. Zinner, S. H., J. Blaser, B. B. Stone, and M. C. Groner. 1985. Use of an in-vitro kinetic model to study antibiotic combinations. J. Antimicrob. Chemother. 15(Suppl. A):221-226. 